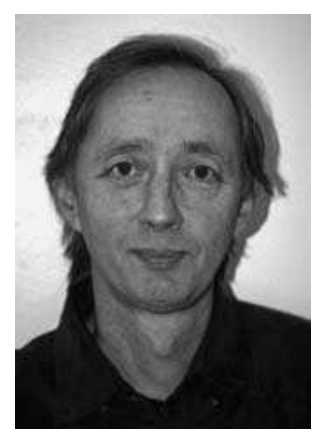

\title{
Quills, Wills and Ills of the Same Feather
}

This issue of LINKS contains a number of bird cases, and reports some triturations of bird-derived substances. Leaving aside all discussion as to what is or is not acceptable to "tradition", the material presented in this edition of LINKS is the product of applying one simple idea - resonance, "like calling to like", "like knowing like" and, of course, "like curing like" - to a distinct aspect of human experience: by name, that way of being which is summoned by any standard usage of the word (in English) "bird" (and by all comparable usage in all other human languages, as indeed by its usage as symbol). Learning to read such "simple" meanings based on our experience of the real world is a popular approach within our current homeopathic renaissance.

What do birds, provings and triturations have in common? Although particulars vary, each can be related to the concept of the flock, even though in each instance degrees of individuality are retained. A flock of birds moves through the air and retains a high degree of unity, acting as if with one will while formed of individual birds. A proving moves through the medium of the individual provers' lives, attracting to itself (and thereby revealing) whatever resonates with the pattern of the potentised substance.

What is the scientific explanation for the coherence, through time and space, of a flock of birds? Departure of a flock, for example, has been described by one scientist as "a contagious phenomenon", but science currently lacks explanations that do not rely on metaphor. And yet birds, not knowing that they are behaving inexplicably, continue to flock.

As Itzhak Bentov suggested in "Stalking the Wild Pendulum", the "contagiousness" of consciousness is much like putting together several grandfather clocks in the same room. Even though at first the pendulums all swing at different intervals, over time they start to synchronize and eventually become uniform, as if one.

Altered states are equally "contagious". Contemporary science hypothesizes that this outcome arises through the synchronization of brain waves, naming this process "entrainment" or "direct induction". It seems likely that the homeopathic proving and the trituration are examples of this very same "contagion".

Underneath our solid appearance, each one of us is actually a bundle of vibrations, fluctuations of energy. Accordingly, in a group, we all come eventually to pulsate in synchronicity with the most powerful vibration within that group. We all form into one unified field. This fact is utilised by such writers as Belleruth Naparstek, who makes the preceding points in "Staying Well with Guided Imagery". It is also utilised in any homeopathic proving where the remedy's pattern does indeed manage to act as the most powerful vibration - as the strange, or hidden, attractor for the experience of the provers.

A proving group, and a trituration group, form a flock which has a unitary, and/or a simultaneous, existence: "as if one person" as Jeremy Sherr puts it in his dynamic book on provings.

Similarly, the individual patient's pathological state is at one with a pattern, part of an invisible flock (disregarding the bounds of time and space), the nature of which the homeopathic practitioner must determine ("dia-gnose") and then return to nothing (by prescribing the simillimum).

A flock of birds in migration will often proceed in a "V" formation. Birds take turns at leading. The lead bird works at flapping its wings while the others enjoy the undulating wake of turbulence created behind it, those who follow simply allowing the rolling waves to move them up and down and thence forwards. A very simple method...

In "The Conference of Birds", an allegory by Fariduddin Attar ("the Chemist"), thirty birds travel together toward a far destination, seeking the king of birds, the Simurgh. On arriving, they discover that they, the thirty birds themselves, are that which they sought. The journey together has proved their unity. The story teaches the reader how to travel - seeking truth through many levels of meaning, in company, following an exemplar or teacher.

Proving, triturating, flocking, seeking, resonating, unifying, migrating, returning - these are the verbs of homeopathy. Vivified by these same verbs, there are many processes of a like nature with homeopathic practice. These verbs are the birds that lead our flock and certain teachers and their teachings, exemplifying and verifying, embody them at different times and in different places.

This issue of LINKS follows on behind Jonathan Shore's "Birds" and after the work of other contemporary teachers and the many modern provings of bird remedies, from Jeremy Sherr's Eagle (Haliaeetus leucocephalus) onwards.

As guest editor of this issue, I hope that the reader benefits from experiencing each article that follows, and finds him- or herself buoyed up and informed by the whole.

The articles are contributed, in the main, by residents or natives of, or immigrants to, Canada. Some time ago there was a wordplay, in German, on the name "Canada" (keine da! or "nothing there") which, then and now, was as little true of Canada as it is of provings, of triturations and of homeopathy itself. Accordingly, this issue of LINKS declares, along with every other issue, "Even so, we're here!"

lain Marrs, Guest Editor 